Trindade, I. A., Ferreira, C., \& Pinto-Gouveia, J. (2018). The longitudinal effects of emotion regulation on physical and psychological health: A latent growth analysis exploring the role of cognitive fusion in IBD. British Journal of Health Psychology, 1(23), 171-185. doi: 10.1111/bjhp.12280

The final publication is available at Wiley via http://onlinelibrary.wiley.com/doi/10.1111/bjhp.12280/abstract 
The longitudinal effects of emotion regulation on physical and psychological health: A latent growth analysis exploring the role of cognitive fusion in IBD

Inês A. Trindade, MSc, PhD student $1 *$ Cláudia Ferreira, $\mathrm{PhD}^{1}$ José Pinto-Gouveia, $\mathrm{MD}, \mathrm{PhD}^{1}$

${ }^{1}$ CINEICC - Cognitive and Behavioural Centre for Research and Intervention Faculty of Psychology and Education Sciences University of Coimbra, Portugal

*Corresponding author.

Inês A. Trindade, Faculdade de Psicologia e de Ciências da Educação da Universidade de Coimbra, Rua do Colégio Novo, 3000-115 Coimbra, Portugal.

Email: ines.almeidatrindade@gmail.com 


\title{
The longitudinal effects of emotion regulation on physical and psychological health: A latent growth analysis exploring the role of cognitive fusion in IBD
}

\begin{abstract}
Objective. This study thus aims to test differences between patients with inflammatory bowel disease (IBD) regarding IBD symptomatology, cognitive fusion, and psychological and physical health, as well as to explore whether the maladaptive emotion regulation process of cognitive fusion longitudinally impacts on the baseline and evolution of these outcomes over a period of 18 months.
\end{abstract}

Design and methods. Participants include 116 IBD patients with a mean age of 36.76 $(S D=11.39)$ of both genders $(69.83 \%$ females $)$ that completed the self-report measures of interest in three different times, equally spaced 9 months apart, over a period of 18 months. Latent growth curve models were conducted using structural equation modelling to estimate the growth trajectory of the variables in study.

Results. Inflammatory bowel disease symptomatology and cognitive fusion's levels were negatively associated with psychological health and physical health's baseline levels. Furthermore, IBD symptomatology did not influence the growth of psychological health, while cognitive fusion did $(b=.30, p=.007)$. The same result was found for physical health $(b=.26, p=.024)$. These findings indicate that individuals with higher levels of cognitive fusion present lower levels of psychological health and physical health that tend to further decrease over the time through the effects of this maladaptive emotion regulation process.

Conclusions. This study implies that it is of crucial importance to include psychotherapeutic interventions in the health care of patients with IBD. If successful, 
these interventions could represent decreases in the cost of IBD treatment and in the use of drugs with adverse side effects, in addition to improving patients' mental health and quality of life. Further implications for clinical and research work are discussed.

Keywords: emotion regulation; cognitive fusion; psychological health; physical health; inflammatory bowel disease; latent growth modeling. 


\section{Introduction}

Emotion regulation refers to the processes by which individuals influence and shape one's emotions and how one experiences and displays those emotions (Gross, 1998). It involves the goal to modify the emotion-generative process and the engagement in processes that can alter the emotion trajectory (Gross, Sheppes, \& Urry, 2011). Emotion regulation can thus range from conscious efforts to initiate, inhibit, or modulate one's internal experiences (such as feelings, thoughts, and emotion-related physiological responses) in a given situation to unconscious automatic regulation of those experiences (Gyurak, Gross, \& Etkin, 2011). Emotion regulation can increase or decrease the latency, frequency, intensity, and duration of an emotional response, but not always as desired by the individual (Gross, 1998), and is so motivated to decrease negative emotions and increase positive ones (Hochschild, 1983; Langston, 1994). Nevertheless, some types of emotion regulation present paradoxical consequences by increasing aversive emotional states one was trying to regulate (Hayes, Strosahl, \& Wilson, 2012). The type of emotion regulation one uses to deal with internal experiences presents therefore a significant influence on one's well-being. Indeed, previous studies have shown that maladaptive forms of emotion regulation are linked to decreased psychological and physical health (e.g., Gross, 2002; Salovey, Rothman, Detweiler, \& Steward, 2000; Segerstrom, Stanton, Alden, \& Shortridge, 2003; Song et al., 2015; van Heck \& den Oudsten, 2008). These findings illustrate the relevant association between emotion regulation and health, and by specifically comprising physical health outcomes, argue against the popular, traditionalist stance of the mind-body dualism (Descartes, 1985 [1641]).

One way of regulating internal experiences is becoming fused with them. Cognitive fusion is considered a maladaptive emotion regulation process, key to the Acceptance and Commitment Therapy psychological inflexibility model (Hayes et al., 
2012). This process can be defined as the tendency to get caught up with thoughts' verbal content, that is, to consider thoughts, as trustworthy interpretations of reality, instead of transient and subjective internal experiences (which ability relates to cognitive defusion). Cognitive fusion is related but theoretically different than rumination. While rumination is described as self-focused repetitive thinking on personal negative feelings and selfreflection on the negative events that have triggered these feelings (Nolen-Hoeksema, Wisco, \& Lyubomirsky, 2008), cognitive fusion relates to the excessive attachment with the literal meaning of their internal experiences instead of viewing them as transient processes. As the individual gets entangled with his or her difficult or aversive thoughts, cognitive events tend to overly influence behaviour and the range of possible actions is consequently limited (Hayes et al., 2012). One's behavioural repertoire is thus retrained by the dominance of internal experiences over other sources of behavioural regulation. This can be exemplified by fusion with thoughts such as 'If I go out, I will feel embarrassed' which may subsequently lead to avoidance patterns of behaviour which aim to control the intensity or frequency of internal experiences, usually uncomfortable ones (e.g., embarrassment, shame, anxiety). Limitations in the range of possible behaviours usually restrain the individual from living a valued, engaged, and full life (Hayes et al., 2012), leading to diminished life satisfaction and decreased quality of life (e.g., Trompetter et al., 2013). Cognitive fusion's link with lower levels of mental health is fairly well established. Several studies point out the association between this process and decreased quality of life and mental health (e.g., Ferreira \& Trindade, 2015; Gillanders et al., 2014; Trindade \& Ferreira, 2014). Nonetheless, to our current knowledge, only one study has explored the causal, longitudinal role of cognitive fusion in mental health. This study (Trindade, Ferreira, \& Pinto-Gouveia, 2017) has namely revealed the impact of cognitive fusion and rumination on depressed mood measured one and a half year later, 
in a sample of patients with inflammatory bowel disease (IBD). This study seems to establish the impact of cognitive fusion on psychopathology in IBD, a condition often considered as lacking empirical support regarding the effects of associated psychological mechanisms on patients' diminished quality of life and clinical evolution (e.g., McCombie, Mulder, \& Gearry, 2013; Sajadinejad, Asgari, Molavi, Kalantari, \& Adibi, 2012).

Inflammatory bowel disease seems to be a much deserving population for studying these aspects due to its adverse symptomatology (abdominal pain, persisting diarrhoea, rectal bleeding, loss of appetite, weight loss, and fatigue), caused by a chronic, auto-immune, and relapsing inflammation of the intestinal system and consequences on quality of life and mental health. The two most common types of IBD are Crohn's disease (CD) and ulcerative colitis (UC), and both illnesses and related symptomatology impair patients' psychosocial functioning and health-related quality of life. It has been demonstrated that IBD may affect all of patients' life domains, from work and household tasks to the quality of intimate relationships (Graff et al., 2006; Irvine, 2008). Even during periods of remission, patients' wellbeing and psychological functioning tend to be poorer in comparison with the normal population (Blondel-Kucharski et al., 2001; Graff et al., 2009). Impaired quality of life in IBD is associated with more relapses and additional medical complications, intensifying the disease process in a cycle of inflammation and psychological suffering (e.g., Levenstein, 2004; Simren et al., 2002).

There is thus much pertinence in investigating the link between IBD-related experiences and decreased well-being. It has been considered that the impact of difficult experiences (such as having IBD) on quality of life may depend on the way one deals with these difficulties (Segal, Williams, \& Teasdale, 2002). In this line, it can be hypothesized that different patients, with different patterns of emotion regulation, may 
present divergences in the way the same illness impacts their well-being. Further, as previous literature has demonstrated the impact of emotion regulation on physical outcomes (e.g., Salovey et al., 2000; Song et al., 2015; van Heck \& den Oudsten, 2008), it can also be hypothesized that cognitive fusion may present a negative impact on patients' physical functioning. Nevertheless, these assumptions lack empirical support.

This study thus aims to further expand previous work on the impact of emotion regulation on health outcomes in IBD (Trindade et al., 2017) by testing differences between patients with IBD regarding IBD symptomatology, cognitive fusion, and psychological and physical health, as well as exploring whether cognitive fusion longitudinally impacts on the evolution of these outcomes. We expect that the variables will not present significant changes over time and that both IBD symptomatology and cognitive fusion will influence the baseline and growth levels of psychological and physical health; and that cognitive fusion will be a stronger predictor of these outcomes.

\section{Materials and Methods}

\section{Setting and participants}

Participants were recruited through the Portuguese Association for IBD (APDI), which emailed its members registered as patients (556 members) with an invitation to participate in this investigation. Patients were informed about the voluntary character of the study and the confidentiality of their responses and personal data. Patients who agreed to participate gave their written informed consent and were asked to complete an online survey with self-report measures, in three different waves of assessment equally spaced by 9 months. Data collection thus took place for 18 months, from the end of 2014 to the 
beginning of 2016. The study was approved by the ethics committees of all involved institutions (FPCE-UC; APDI).

Two hundred and nine (209) participants completed Wave 1, 168 participants (80.38\%) completed Wave 2, and 127 participants (60.77\%) completed Wave 3. Only, the participants who completed the three waves of assessment $(\mathrm{N}=123 ; 58.85 \%)$ were considered be included in the current study. Furthermore, from this pool of 123 participants, seven $(5.69 \%)$ were excluded for reporting other physical illnesses (breast cancer, fibromyalgia, thalassaemia, multiple sclerosis, diabetes) or psychiatric disorders (bipolar disorder, generalized anxiety disorder).

The sample of this study thereforecomprises 116 patients with IBD of both genders: 70 patients (60.34\%) diagnosed with Crohn's disease, 43 (37.07\%) with ulcerative colitis, and three (2.59\%) with IBD-unknown. Table 1 further presents the descriptive statistics of the sample.

Please Insert Table 1 around here

\section{Measures}

The online survey included demographic and medical history questions - gender, age, completed level of education, marital status, form of IBD, time since IBD diagnosis, and the occurrence of IBD symptoms during the previous month (10 symptoms measured on a self-report 6-point scale (0: Never; 6: Always): fatigue, abdominal pain, excessive gas, difficulties in gaining or maintaining weight, tenesmus, diarrhoea, nausea, bloody stools, abdominal distension, fever). This scale was specially created for this study to allow the assessment the frequency of the experience of specific IBD symptoms; a total summed score was used,which reflects the level of occurrence of IBD symptomatology 
during the previous month. Furthermore, participants filled the Portuguese validated versions of the following questionnaires in each wave of assessment:

Cognitive Fusion Questionnaire-7 (Portuguese validation by Costa, Mar^oco, \& PintoGouveia, 2017; CFQ-7; Gillanders et al., 2014)

This 7-item instrument was developed to assess one's level of cognitive fusion (e.g., 'It's such a struggle to let go of upsetting thoughts even when I know that letting go would be helpful'). It is rated on a 7-point scale from 1: Never true to 7: Always true. The CFQ-7 has presented very good psychometric characteristics in its original (as between .88 and .93 across five samples) and Portuguese (as between .89 and .94 across three samples) validation studies.

World Health Organization Brief Quality of Life Assessment Scale (Portuguese validation by Canavarro et al., 2009; WHOQOL-BREF; WHOQOL Group, 1998)

This 26-item scale aims to assess one's subjective perception of their QoL, in four different domains (physical health, psychological health, social relationships, and environment). Only two domains were used in this study: physical health (e.g., 'To what extent do you feel that physical pain prevents you from doing what you need to do'; 'Do you have enough energy for everyday life?'; 'How satisfied are you with your sleep?') and psychological health ('How much do you enjoy life?'; 'Towhat extent do you feel your life to be meaningful?'; 'How often do you have negative feelings such as blue mood, despair, anxiety, depression?'). Items are assessed using a 5-point Likert scale, with higher scores indicating higher levels of perceived QoL. The WHOQOL-BREF presented psychometric adequacy in the original (as between .66 and .84 ) and Portuguese (as between .67 and .87) validation studies. 
The Cronbach's alphas presented by these instruments in the current study are presented in Table 2.

\section{Statistical Analysis}

Firstly, preliminary data analyses were conducted to test the adequacy of the data for further analysis. Descriptive and frequency analyses were performed to analyse the sample's demographic and medical features. Furthermore, the relationships between the variables in study were examined through Pearson's correlation coefficients (Cohen, Cohen, West, \& Aiken, 2003). These analyses were conducted using SPSS, version 22.0 (IBM Corp, 2013).

Latent growth curve models (LGMs; Willett \& Sayer, 1994; Windle, 1997) were conducted using structural equation modelling (AMOS, version 22.0; Arbuckle, 2013) to estimate the growth trajectory of IBD symptomatology, cognitive fusion, psychological health, and physical health. This is a longitudinal analysis that estimates growth over a period of time (in the case of the current study, 18 months) that approaches the analysis of repeated measures from the perspective of an individual growth curve for each participant. Each growth curve has a certain baseline level (the intercept factor) and a certain rate of change over time (the slope factor). The intercept factor is a constant for any individual across time and thus describes the initial level of the variable in study (intercept mean) and its individual differences (intercept variance). In turn, the slope factor indicates the average rate of change (slope mean) and individual differences in patterns of growth (slope variance). The direction of the correlation between the intercept and slope factors is also analysed: If positive, it indicates that the lower the initial level, 
the higher the growth; if negative, it demonstrates that the larger the baseline, the lower the growth.

In a second step, hypotheses about predictors of the growth of the outcome measures (psychological health and physical health) were tested. To do so, the models of these variables were conditioned by including IBD symptomatology and cognitive fusion as predictors of the intercept and the slope factors. In this way, one can examine whether these predictors account for individual differences in the initial level of the outcomes and for the differences in the rate of change.

To analyse the adequacy of the models, several goodness of fit was examined: the Comparative Fit Index (CFI), the Tucker and Lewis Index (TLI), and the Incremental Fit Index (IFI) which show that the tested model presents a good adjustment to the empirical data when around .95 (Hu \& Bentler, 1999), and the Standardized Root Mean Squared Residual (SRMR) that demonstrates a good model fit when inferior to .08 (Hu \& Bentler, 1999).

\section{Results}

\section{Preliminary analysis}

Skewness and Kurtosis' values indicated that the variables in study did not present a significant bias to normal distribution (Skewness varied between -.49 and .58 , and Kurtosis between -.63 and .39). The visual inspection of distributions further confirmed the assumption of normality (Kline, 2005).

Correlation analyses' results are presented in Table 2, and it is worth observing that cognitive fusion at baseline was negatively linked with psychological health and physical 
health at baseline and at Wave 2 (9 months later). Also, cognitive fusion at baseline wa linked to psychological health at Wave 3 (18 months later).

Please Insert Table 2 around here

Non-conditioned models

IBD Symptomatology

The LGM was successfully fitted to the levels of IBD symptomatology physical health's at the three waves of assessment: CFI $=1.00 ; \mathrm{TLI}=1.00 ; \mathrm{IFI}=1.00 ; \mathrm{SRMR}=$ .00 .

Basal levels of IBD symptomatology were significantly different among participants $(b=61.57 ; S E=13.31 ; Z=24.66 ; p<.001)$ around a mean of $21.21(S E=$ $0.88 ; Z=24.06 ; p<.001)$. Slope's mean indicated a non-significant general change over time $(b=-0.51 ; S E=0.33 ; Z=-1.57 ; p=.117)$. Furthermore, the growth rate of IBD symptomatology was homogeneous among participants given the non-significant variance around the mean growth $(b=-2.15 ; \mathrm{SE}=5.58 ; \mathrm{Z}=-0.39 ; p=.700)$.

\section{Cognitive Fusion}

The LGM was successfully fitted to cognitive fusion levels assessed at the three waves, as indicated by the excellent model fit: $\mathrm{CFI}=.98 ; \mathrm{TLI}=.95 ; \mathrm{IFI}=.98 ;$ SRMR $=$ .00 .

Basal levels of cognitive fusion presented individual differences between participants $(b=80.37 ; S E=14.80 ; Z=5.43 ; p<.001)$ around a mean level of $22.29(S E$ $=0.94 ; Z=23.74 ; p<.001)$. Overall, there was no significant change over time as 
indicated by the non-significant estimate of slope's mean $(b=-0.64 ; S E=0.42 ; Z=-1.51$; $p=.131$ ). The growth rate of cognitive fusion was homogeneous among participants given the non-significant variance around the mean growth $(b=10.76 ; S E=5.71 ; Z=$ $1.88 ; p=.060)$. The correlation between intercept and slope was $-.17(p=.434)$.

Psychological health

The LGM was adequately fitted to psychological health's levels at the three waves of assessment: $\mathrm{CFI}=.97 ; \mathrm{TLI}=.91 ; \mathrm{IFI}=.97 ; \mathrm{SRMR}=.00$.

Initial levels of psychological health presented individual differences among participants $(b=215.08 ; S E=40.95 ; Z=5.25 ; p<.001)$ around a mean level of 64.05 $(S E=1.44 ; Z=44.29 ; p<.001)$. Slope's mean was non-significant $(b=1.19 ; S E=0.64$ $Z=1.84 ; p=.065)$, indicating that there was no significant change over time regarding psychological health's levels. The growth rate was not homogeneous among participants as shown by the significant variance around the mean growth $(b=37.21 ; S E=14.47 ; Z$ $=2.57 ; p=.010)$. The association between intercept and slope was $-0.42(p=.040)$, indicating that the larger the level of psychological health at baseline, the lower the subsequent rate of change.

Physical health

The LGM was successfully fitted to physical health's levels measured at the three waves: $\mathrm{CFI}=1.00 ; \mathrm{TLI}=1.00 ; \mathrm{IFI}=1.00 ; \mathrm{SRMR}=.00$.

Basal levels of physical health were significantly different among participants $(b$ $=256.75 ; S E=47.77 ; Z=5.38 ; p<.001)$ around a mean level of $63.62(S E=1.58 ; Z=$ 40.16; $p<.001)$. Slope's mean was found to be significant, indicating a significant tendency to increase at a mean rate of $1.53 / \mathrm{yr}(S E=0.69 ; Z=2.22 ; p=.026)$. Further, the 
growth rate was not homogeneous among participants as revealed by the significant variance around the mean growth $(b=36.30 ; S E=16.55 ; Z=2.19 ; p=.028)$. The correlation between intercept and slope was $-0.46(p=.037)$, showing that the higher the level of physical health at baseline, the lower the later rate of change.

\section{Predicting change}

Psychological health

A conditional model was fitted to data to explore the influence of IBD symptomatology and cognitive fusion on the growth of psychological health (Figure 1). This model presented a very good fit to the empirical data: $\mathrm{CFI}=.98 ; \mathrm{TLI}=.94 ; \mathrm{IFI}=$ $.98 ; \mathrm{SRMR}=.01$.

Results showed that IBD symptomatology presented a significant impact on psychological health baseline levels $(b=-.29, p<.001)$, indicating that individuals who report more symptomatology present lower levels of psychological health. Nevertheless, it was also shown that IBD symptomatology did not influence the growth rate of psychological health $(b=.15, p=.169)$.

Regarding cognitive fusion, it was shown that this process significantly impacted on psychological health baseline levels with a negative effect of $-0.62(p<.001)$. Furthermore, the rate of psychological health growth was significantly influenced by cognitive fusion $(b=.30, p=.007)$.

Physical health

Similar results were found for physical health. This conditional model (Figure 2) was fitted to data to explore the influence of IBD symptomatology and cognitive fusion 
on the growth of physical health and showed an excellent fit: CFI $=1.00 ; \mathrm{TLI}=1.00 ; \mathrm{IFI}$ $=1.00 ; \mathrm{SRMR}=.01$.

The model indicated that IBD symptomatology had a significant negative effect on basal physical health levels $(b=-.59, p<.001)$, demonstrating that individuals who report more IBD symptomatology show lower levels of physical health. However, IBD symptomatology did not present a significant effect on the growth rate of physical health $(b=.15, p=.206)$; that is, IBD symptomatology also did not predict individual differences in the evolution of physical health.

Additionally, results showed that cognitive fusion had a significant negative effect on the baseline levels of physical health $(b=-.28 ; p<.001)$. Moreover, the rate of physical health growth was significantly impacted by cognitive fusion $(b=.26, p=.024)$.

\section{Discussion}

This study examined the progression of IBD symptomatology, cognitive fusion, and psychological and physical health in a sample of patients with IBD, over a time period of 18 months with three assessment moments. Also, we intended to examine the association of IBD symptomatology and cognitive fusion with psychological health and physical health, and explore whether IBD symptomatology and cognitive fusion significantly predict longitudinal changes in these outcomes.

Latent growth curve models results revealed that IBD symptomatology and cognitive fusion's initial levels presented significant differences among participants and that these variables did not present overall significant changes over time. These findings indicate that participants reported different levels of IBD symptomatology and cognitive fusion and also that these variables seem to be fairly stable over time. Results also 
demonstrated that the change rate of IBD symptomatology and cognitive fusion did not differ significantly among participants.

Regarding psychological health, the LGM analysis showed that its initial levels also differed among participants and that there was no overall change in this variable over the assessed 18 months. Psychological health in patients with IBD thus seems to be generally stable over time. Nevertheless, the change rate of psychological health was demonstrated to differ among participants: Some participants presented similar levels over time, while others' levels increased, and other participants showed decreased psychological health over time. Individuals who presented higher levels at baseline presented a smaller progression over time in psychological health. Similar results were found for physical health with the exception of its overall change over time. Physical health was found to present a tendency to increase over the measured 18 months.

Taking into consideration these data, the clinical features of IBD, and previous considerations regarding the effects of emotion regulation on health (e.g., Gross, 2002; Salovey et al., 2000; Segerstrom et al., 2003; Song et al., 2015; van Heck \& den Oudsten, 2008), two conditional LGMs were tested to investigate the influence of IBD symptomatology and cognitive fusion on the evolution of patients' psychological and physical health over 18 months. Concerning psychological health, it was revealed that both IBD symptomatology and cognitive fusion's levels were negatively associated with psychological health's baseline levels. Furthermore, results indicated that IBD symptomatology did not influence the growth of psychological health, while cognitive fusion did. Findings seem to be particularly relevant by thus indicating that individuals with higher levels of cognitive fusion present lower levels of psychological health that tend to further decrease through the effects of this maladaptive emotion regulation process. 
These findings go in line with previous studies demonstrating the effects of coping strategies (e.g., illness perceptions) on self-reported disability (van der Have et al., 2015) and adjustment to the disease (Dorrian, Dempster, \& Adair, 2009) in patients with IBD. Our data also are in line with previous research demonstrating the association between cognitive fusion and decreased mental health (e.g., Ferreira \& Trindade, 2015; Gillanders et al., 2014; Trindade \& Ferreira, 2014). These results seem to suggest that cognitive fusion, described as the dominance of verbal relations over direct experience in controlling behaviour, is related to overthinking and lack of action (Gillanders et al., 2014; Hayes et al., 2012), which may ultimately lead to diminished psychological health. Furthermore, as cognitive fusion is linked with other maladaptive processes of the ACT's psychological inflexibility model, such as life values confusion, uncommitted action, and experiential avoidance (Hayes et al., 2012), it may be possible that these interactions further expand and explain cognitive fusion's detrimental effect on mental functioning. For instance, if fused with thoughts such as 'I can't go out with my friends, because my illness will get in the way' or 'This illness is so embarrassing I can't talk to anyone about it', a patient may tend to avoid activities he actually values and enjoys (e.g., having quality time with friends/family; having close and secure social relationships). Like so, one may rather act uncommittedly with life values and objectives, which can lead to a sense of life unfulfillment and isolation.

The current study also aimed to explore the influence of IBD symptomatology and cognitive fusion on the physical health of patients with IBD. Results indicated that the baseline levels of IBD symptomatology and cognitive fusion were inversely linked with physical health. Also similarly to what was found in the psychological health analysis, it was revealed that IBD symptomatology did not influence the evolution of physical health, while cognitive fusion did present significant effects on this outcome. Thus, IBD patients 
with higher levels of cognitive fusion present lower levels of physical health that tend to further decline with the influence of this emotion regulation process.

These findings can be explained under the framework of several models regarding the link between emotion regulation and physical health. According to the transactional/ interactional stress-illness model (van Heck\&den Oudsten, 2008), emotion regulation, by determining the selection, manipulation, and evaluation of difficult events, modulates physiological responses to stressors. In this line, results from the current study indicate that cognitive fusion may amplify the perceived importance and meaning of unwanted/ difficult internal experiences, augmenting the frequency and intensity of related negative emotions, and thus impacting on physiological levels. When facing disease-related stressful situations, patients with IBD may get fused with difficult thoughts about their condition, symptoms, or clinical evolution, which expectedly leads to negative affect and to the further occurrence of negative internal experiences (Hayes et al., 2012). One may hypothesize that the link between cognitive fusion and subsequent depressed mood, found in patients with IBD (Trindade et al., 2017), may similarly explain the impact of cognitive fusion on physical health. Given that depressed mood can stimulate the production of proinflammatory cytokines, modulating the clinical expression of IBD (Kiecolt-Glaser, McGuire, Robles, \& Glaser, 2002; Tache \& Bernstein, 2009), high depressive levels may thus lead to diminished physical health in patients with IBD. Along these lines, cognitive fusion's depressenogenic effects may explain its impact on physical health.

Furthermore, the health behaviour model proposes that emotion regulation influences health-related behaviours and coping behaviours (e.g., eating healthy, taking the prescribed medications), which mediate the link between emotion regulation and physical health (van Heck \& den Oudsten, 2008). Accordingly, it can be suggested that patients with IBD, when fusedwith thoughts regarding their perception of the illness and 
their current health may engage in health-degrading habits. For example, if a patient gets entangled with thoughts such as 'I'm always going to be this sick', 'diet won't impact my health', or 'medication only makes me feel worse', he or she may tend to neglect medical recommendations, and present poor adherence to medication/diet and attendance to medical appointments.

These findings present nonetheless some limitations. The sample size and dropout impose some caution in the interpretation of the findings. Larger samples are preferable to conduct SEM models (e.g., Wolf, Harrington, Clark, \& Miller, 2013); nevertheless, it is generally recommended that LGM analyses should be conducted using samples of at least 100 (Hamilton, Gagne, \& Hancock, 2003), which is the case of the current study. Further, the sample comprises Portuguese patients who were recruited through an association via an email invitation. Future research should confirm this study's findings using samples of other cultures and recruited by different methods (e.g., in hospital settings). The self-report nature of this study may also represent a methodological limitation; future studies ought to comprise laboratorial medical indices and clinical interviews to assess psychological and physical health. The longitudinal analysis of the effects of other emotion regulation processes such as acceptance and mindfulness on health could also be of interest. Nevertheless, this study provides important novel data regarding the longitudinal impact of emotion regulation, namely cognitive fusion, on psychological and also physical health outcomes in IBD.

The present study indeed may suggest that promoting adaptive emotion regulation strategies may improve IBD patients' psychological and physical health. Future studies should nonetheless attempt to repeat this study's findings with larger samples to support the development of psychotherapeutic interventions for patients with IBD. If this study's results are corroborated, ACT-based interventions would probably be helpful to provide 
effective results due to their focus on the promotion of defusion and acceptance abilities and committed action. These interventions could represent decreases in the cost of IBD treatment and in the use of drugs with adverse side effects, in addition to improving patients' mental health and quality of life.

\section{Acknowledgements}

We are grateful for the help provided by the Portuguese Association for IBD (Associação Portuguesa para a Doença Inflamatória do Intestino; APDI) during the recruitment process of the current research project. We would also like to thank Dr. Cristiana Duarte for her help during the conduction of the LGM analysis.

\section{Funding}

Research by Inês A. Trindade is supported by a Ph.D. Grant (SFRH/BD/101906/2014) sponsored by FCT (Portuguese Foundation for Science and Technology).

\section{References}

Arbuckle, J. L. (2013). Amos 22.0 user's guide. Chicago, IL: IBM SPSS.

Blondel-Kucharski, F., Chircop, C., Marquis, P., Corot, A., Baron, F., Gendre, J. P., . . . Groupend'Etudes Therapeutique des Affections Inflammatoires Digestives (2001). Health-related qualitynof life in Crohn's disease: A prospective longitudinal study in 231 patients. American Journal of Gastroenterology, 98, 2915-2920. https://doi.org/10.1016/s0002-9270(01)03243-9 
Canavarro, M. C., Serra, A. V., Simoes, M. R., Rijo, D., Pereira, M., Gameiro, S., . . . Paredes, T. (2009). Development and psychometric properties of the World Health Organization Quality of Life Assessment Instrument (WHOQOL-100) in Portugal. International Journal of Behavioral Medicine, 16, 116-124. https://doi.org/10.1007/s12529-008-9024-2

Cohen, J., Cohen, P., West, S.,\&Aiken, L. (2003). Applied multiple regression/correlation analysis for the behavioral sciences (3rd ed.). Mahwah, NJ: Lawrence Erlbaum.

Costa, J. A., Marôco, J., \& Pinto-Gouveia, J. (2017). Validation of the psychometric properties of cognitive fusion questionnaire. A study of the factorial validity and factorial invariance of the measure among osteoarticular disease, diabetes mellitus, obesity, depressive disorder, and general populations. Clinical Psychology \& Psychotherapy. Advanced online publication. https://doi.org/10.1002/cpp.2077

Descartes, R. (1985 [1641]). Meditations on first philosophy. In J. Cottingham, R. Stoothoff \& D. Murdoch (Eds.), The Philosophical writings of descartes. Cambridge, UK: Cambridge University Press.

Dorrian, A., Dempster, M., Adair, P. (2009). Adjustment to inflammatory bowel disease: The relative influence of illness perceptions and coping. Inflammatory Bowel Diseases, 15(1), 47-55. https://doi.org/10.1002/ibd.20583

Ferreira, C., \& Trindade, I. A. (2015). Body image-related cognitive fusion as a main mediational process between body-related experiences and women's quality of life. Eating and Weight Disorders, 20(1), 91-97. https://doi.org/10.1007/s40519- 014$0155-\mathrm{y}$

Gillanders, D. T., Bolderston, H., Bond, F. W., Dempster, M., Flaxman, P. E., Campbell, L., . . Remington, B. (2014). The development and initial validation of The 
Cognitive Fusion Questionnaire. Behavior Therapy, 45, 83-101. https://doi.org/10.1016/j.beth.2013.09.001

Graff, L. A., Walker, J. R., \& Bernstein, C. N. (2009). Depression and anxiety in inflammatory bowel disease: A review of comorbidity and management. Inflammatory Bowel Diseases, 14, 1105-1118. https://doi.org/10.1002/ibd.20873

Graff, L. A., Walker, J. R., Lix, L. M., Clara, I., Rawsthorne, P., Rogala, L., . . Bernstein, C. N. (2006). The relationship of inflammatory bowel disease type and activity to psychological functioning and quality of life. Clinical Gastroenterology and Hepatology, 4, 1491-1501. https://doi.org/10.1016/j.cgh.2006.09.027

Gross, J. J. (1998). The emerging field of emotion regulation: An integrative review. Review of General Psychology, 2, 271-299.

Gross, J. J. (2002). Emotion regulation: Affective, cognitive, and social consequences. Psychophysiology, 39(3), 281-291. https://doi.org/10.1017/S0048577201393198

Gross, J. J., Sheppes, G., \& Urry, H. L. (2011). Emotion generation and emotion regulation: A distinction we should make (carefully). Cognition \& Emotion, 25, 765-781. https://doi.org/10.1080/02699931.2011.555753

Gyurak, A., Gross, J. J., \& Etkin, A. (2011). Explicit and implicit emotion regulation: A dual-process framework. Cognition \& Emotion, 25, 400-412. https://doi.org/10.1080/02699931.2010.544160

Hamilton, J., Gagne, P. E., \& Hancock, G. R. (2003). The Effect of Sample Size on Latent Growth Models. Annual Meeting of the American Educational Research Association in Chicago, IL

Hayes, S. C., Strosahl, K. D., \& Wilson, K. G. (2012). Acceptance and commitment therapy: The process and practice of mindful change (2nd ed.). New York, NY: The Guilford Press. 
Hochschild, A. R. (1983). The managed heart: Commercialization of human feeling. Berkeley, CA: University of California Press.

Hu, L., \& Bentler, P. M. (1999). Cutoff criteria for fit indexes in covariance structure analysis: Conventional criteria versus new alternatives. Structural Equation Modeling: $\quad A \quad$ Multidisciplinary $\quad J o u r n a l, \quad 6(1), \quad 1-55$. https://doi.org/10.1080/10705519909540118

IBM Corp. Released 2013. IBM SPSS statistics for windows, Version 22.0 [Computer software]. Armonk, NY: IBM Corp.

Irvine, E. J. (2008). Quality of life of patients with ulcerative colitis: Past, present, and future. Inflammatory Bowel Diseases, 14, 554-565. https://doi.org/10.1002/ibd.20301

Kiecolt-Glaser, J. K., McGuire, L., Robles, T. F., \& Glaser, R. (2002). Psychoneuroimmunology: Psychological influences on immune function and health. Journal of Consulting and Clinical Psychology, 70, 537-547. https://doi.org/10.1037/0022-006X.70.3.537

Kline, R. B. (2005). Principles and practice of structural equation modeling (2nd ed.). New York, NY: The Guilford Press.

Langston, C. A. (1994). Capitalizing on and coping with daily-life events: Expressive responses to positive events. Journal of Personality and Social Psychology, 67, $11-12$.

Levenstein, S. (2004). Embracing complexity: What determines quality of life in inflammatory bowel disease? European Journal of Gastroenterology \& Hepatology, 14, 1253-1255. https://doi.org/10.1097/00042737-20041200000002 
McCombie, A. M., Mulder, R. T., \& Gearry, R. B. (2013). How IBD patients cope with IBD: A review. Journal of Crohn's and Colitis, 7, 89-106. https://doi.org/10.1016/j.crohns.2012.05.021

Nolen-Hoeksema, S., Wisco, B. E.,\&Lyubomirsky, S. (2008). Rethinking rumination. Perspectives on Psychological Science, 3, 400-424. https://doi.org/10.1111/j.1745-6924.2008.00088.x

Sajadinejad, M. S., Asgari, K., Molavi, H., Kalantari, M., \& Adibi, P. (2012). Psychological issues in inflammatory bowel disease: An overview. Gastroenterology Research and Practice, 2012, 1-11. https://doi.org/10.1155/2012/106502

Salovey, P., Rothman, a. J., Detweiler, J. B., \& Steward, W. T. (2000). Emotional states and physical health. The American Psychologist, 55(1), 110-121. https://doi.org/10.1037/0003-066X.55.1.110

Segal, Z. V., Williams, J. M. G., \& Teasdale, J. D. (2002). Mindfulness-based cognitive therapy for depression: A new approach to preventing relapse. New York, NY: Guilford Press.

Segerstrom, S. C., Stanton, A. L., Alden, L. E.,\&Shortridge, B. E. (2003). A multidimensional structure for repetitive thought: What's on your mind, and how, and how much? Journal of Personality and Social Psychology, 85, 909-921. https://doi.org/10.1037/0022-3514.85.5.909

Simrén, M., Axelsson, J., Gillberg, R., Abrahamsson, H., Svedlund, J., \& Bjornsson, E. S. (2002). Quality of life in inflammatory bowel disease in remission: The impact of IBSlike symptoms and associated psychological factors. American Journal of Gastroenterology, 14, 389-396. https://doi.org/10.1111/j.1572-0241.2002.05475.x 
Song, Y., Lu, H., Hu, S., Xu, M., Li, X.,\&Liu, J. (2015). Regulating emotion to improve physical health through the amygdala. Social Cognitive and Affective Neuroscience, 10, 523-530. https://doi.org/10.1093/scan/nsu083

Taché, Y., \& Bernstein, C. N. (2009). Evidence for the role of the brain-gut axis in inflammatory bowel disease: Depression as cause and effect? Gastroenterology, 136, 2058-2061. https://doi.org/10.1053/j.gastro.2009.04.032

Trindade, I. A., \& Ferreira, C. (2014). The impact of body image-related cognitive fusion on eating psychopathology. Eating Behaviors, 15(1), 72-75. https://doi.org/10.1016/j.eatbeh.2013.10.014

Trindade, I. A., Ferreira, C., \& Pinto-Gouveia, J. (2017). An 18-month study of the effects of IBD symptomatology and emotion regulation on depressed mood. International Journal of Colorectal Disease, 32(5), 651-660. https://doi.org/10.1007/s00384017-2774-z

Trompetter, H. R., ten Klooster, P. M., Schreurs, K. M. G., Fledderus, M., Westerhof, G. J., \& Bohlmeijer, E. T. (2013). Measuring values and committed action with the Engaged Living Scale (ELS): Psychometric evaluation in a nonclinical and chronic pain sample. Psychological Assessment, 25, 1235-1246. https://doi.org/10.1037/a0033813

van der Have, M., Fidder, H. H., Leenders, M., Kaptein, A. A., van der Valk, M. E., van Bodegraven, A. A., . . Oldenburg, B. (2015). Self-reported disability in patients with inflammatory Bowel disease largely determined by disease activity and illness perceptions. Inflammatory Bowel Diseases, 21(2), 369-377. https://doi.org/10.1097/MIB.0000000000000278

van Heck, G. L.,\&den Oudsten, B. L. (2008). Emotional Intelligence: Relationships to Stress, Health, and Well-being. In A. Vingerhoets, I. Nyklicek \& J. Denollet 
(Eds.), Emotion regulation: Conceptual and clinical issues. New York, NY: Springer.

WHO (1998). Development of the World Health Organization WHOQOL-BREF quality of life assessment. The WHOQOL Group. Psychological Medicine, 28, 551-558. https://doi.org/10.1017/S0033291798006667

Willett, J. B., \& Sayer, A. G. (1994). Using covariance structure analysis to detect correlates and predictors of individual change over time. Psychological Bulletin, 116, 363-381. https://doi.org/10.1037/0033-2909.116.2.363

Windle, M. (1997). Alternative latent-variable approaches to modeling change in adolescent alcohol involvement. In K. J. Bryant, S. G. West \& M. Windle (Eds.), The science of prevention: Methodological advances from alcohol and substance abuse research (pp. 43-78). Washington, DC: American Psychological Association.

Wolf, E. J., Harrington, K. M., Clark, S. L., \& Miller, M. W. (2013). Sample size requirements for structural equation models: An evaluation of power, bias, and solution propriety. Educational and Psychological Measurement, 76, 913-934. https://doi.org/10.1177/0013164413495237 
Table 1. Descriptive statistics and Cronbach's alphas $(N=116)$

\begin{tabular}{|c|c|c|c|c|}
\hline \multirow{2}{*}{\multicolumn{2}{|c|}{ Demographic and medical data at Wave 1}} & $n$ & $M$ & \multirow[t]{2}{*}{$S D$} \\
\hline & & & & \\
\hline \multirow{2}{*}{ Gender } & Male & $35(30.17 \%)$ & & \\
\hline & Female & $81(69.83 \%)$ & & \\
\hline \multicolumn{2}{|c|}{ Age (range: $18-75)$} & & 36.76 & 11.39 \\
\hline \multicolumn{2}{|c|}{ Education (range: $7^{\text {th }}$ grade $-\mathrm{PhD}$ ) } & & 14.75 & 2.74 \\
\hline \multirow{4}{*}{ Marital status } & Single & $54(46.55 \%)$ & & \\
\hline & Married or Cohabitating & $54(46.55 \%)$ & & \\
\hline & Divorced & $7(6.03 \%)$ & & \\
\hline & Widowed & $1(0.86 \%)$ & & \\
\hline \multirow{3}{*}{ Type of IBD } & Crohn's Disease & $70(60.34 \%)$ & & \\
\hline & Ulcerative Colitis & $43(37.07 \%)$ & & \\
\hline & IBD-unknown & $3(2.59 \%)$ & & \\
\hline \multirow{4}{*}{$\begin{array}{l}\text { Time since } \\
\text { diagnosis }\end{array}$} & Until a year & $5(4.31 \%)$ & \multirow{4}{*}{8.76} & \multirow{4}{*}{6.93} \\
\hline & $1-5$ years & $40(34.48 \%)$ & & \\
\hline & $6-10$ years & $35(30.17 \%)$ & & \\
\hline & $>10$ years & $36(31.03 \%)$ & & \\
\hline
\end{tabular}




\section{Table 2}

Means (M), Standard Deviations (SD), Cronbach's alphas $(\alpha)$ and intercorrelation scores of the study variables in the three waves $(N=116)$

\begin{tabular}{|c|c|c|c|c|c|c|c|c|c|c|c|c|c|c|}
\hline & $M$ & $S D$ & $\alpha$ & 1 & 2 & 3 & 4 & 5 & 6 & 7 & 8 & 9 & 10 & 11 \\
\hline 1. IBD symptoms at $\mathrm{W} 1$ & 21.48 & 10.03 & 0.85 & 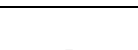 & & & & & & & & & & \\
\hline 2. Cognitive fusion at $\mathrm{W} 1$ & 22.76 & 10.39 & 0.95 & 0.14 & - & & & & & & & & & \\
\hline 3. Psychological health at W1 & 63.76 & 15.63 & 0.80 & $-0.33 * * *$ & $-0.60 * * *$ & - & & & & & & & & \\
\hline 4. Physical health at W1 & 63.58 & 17.14 & 0.84 & $-.058 * * *$ & $-0.38 * * *$ & $0.58 * * *$ & - & & & & & & & \\
\hline 5. IBD symptoms at W2 & 20.41 & 9.63 & 0.84 & $0.71 * * *$ & 0.13 & $-0.27 * *$ & $-0.47 * * *$ & - & & & & & & \\
\hline 6. Cognitive fusion at $\mathrm{W} 2$ & 20.94 & 10.09 & 0.96 & $0.27 * *$ & $0.73 * * *$ & $-0.59 * * *$ & $-0.47 * * *$ & $0.24 * *$ & - & & & & & \\
\hline 7. Psychological health at W2 & 67.28 & 16.41 & 0.85 & $-0.32 * * *$ & $-0.48 * * *$ & $0.70 * * *$ & $0.52 * * *$ & $-0.32 * *$ & $-0.65 * * *$ & - & & & & \\
\hline 8. Physical health at W2 & 65.39 & 17.40 & 0.84 & $-0.54 * * *$ & $-0.22 *$ & $0.42 * * *$ & $0.72 * * *$ & $-0.59 * * *$ & $-0.38 * * *$ & $0.59 * * *$ & - & & & \\
\hline 9. IBD symptoms at W3 & 20.34 & 10.01 & 0.86 & $0.74 * *$ & 0.13 & $-0.28 * *$ & $-0.47 * * *$ & $0.80 * * *$ & $0.24 * *$ & $-0.34 * * *$ & $-0.57 * * *$ & - & & \\
\hline 10. Cognitive fusion at W3 & 21.24 & 10.82 & 0.97 & $0.24 *$ & $0.63 * * *$ & $-0.61 * * *$ & $-0.39 * * *$ & 0.12 & $0.80 * * *$ & $-0.64 * * *$ & $-0.31 * *$ & $0.20 *$ & - & \\
\hline 11. Psychological health at W3 & 66.27 & 15.13 & 0.82 & $-0.21 *$ & $-0.38 * * *$ & $0.60 * * *$ & $0.37 * * *$ & $-0.28 * *$ & $0.55 * * *$ & $0.72 * * *$ & $0.42 * * *$ & $-0.34 * * *$ & $-0.69 * * *$ & - \\
\hline 12. Physical health at W3 & 66.63 & 16.32 & 0.86 & $-0.48 * * *$ & -0.15 & $0.34 * * *$ & $0.61 * * *$ & $-0.49 * * *$ & $0.40 * * *$ & $0.40 * * *$ & $0.70 * * *$ & $-0.62 * * *$ & $-0.31 * *$ & $0.50 * * *$ \\
\hline
\end{tabular}

Note: ${ }^{*} p<0.05 ;{ }^{* *} p<0.01 ;{ }^{* * *} p<0.001$.

$\mathrm{W} 1=$ Wave 1; W2 = Wave 2; W3 = Wave 3. Cognitive fusion measured by the Cognitive Fusion Questionaire-7; Psychological health and Physical health measured by the

World Health Organization Brief Quality of Life Assessment Scale. 


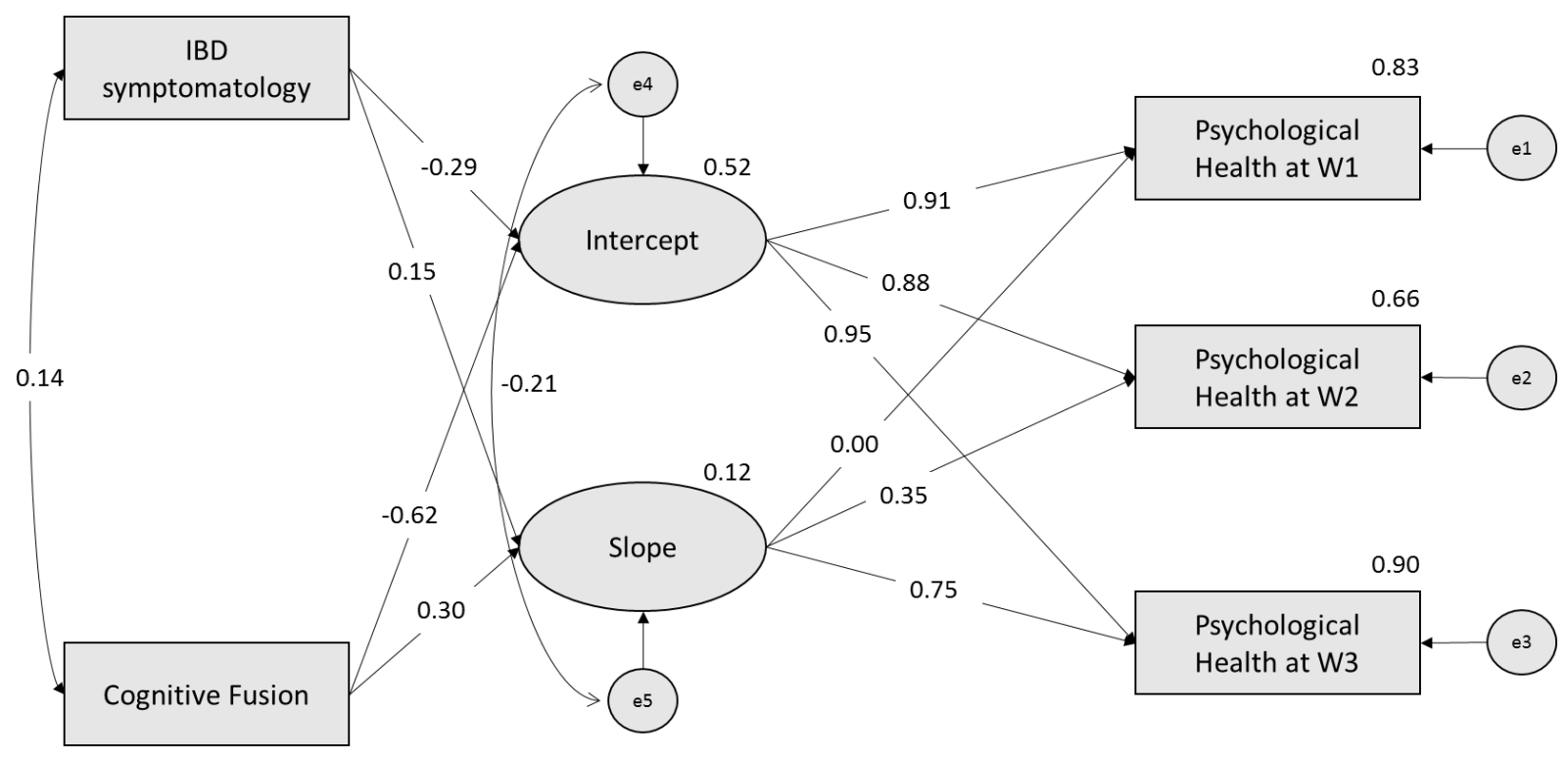

Figure 1. The influence of IBD symptomatology and cognitive fusion in changes in psychological health. 


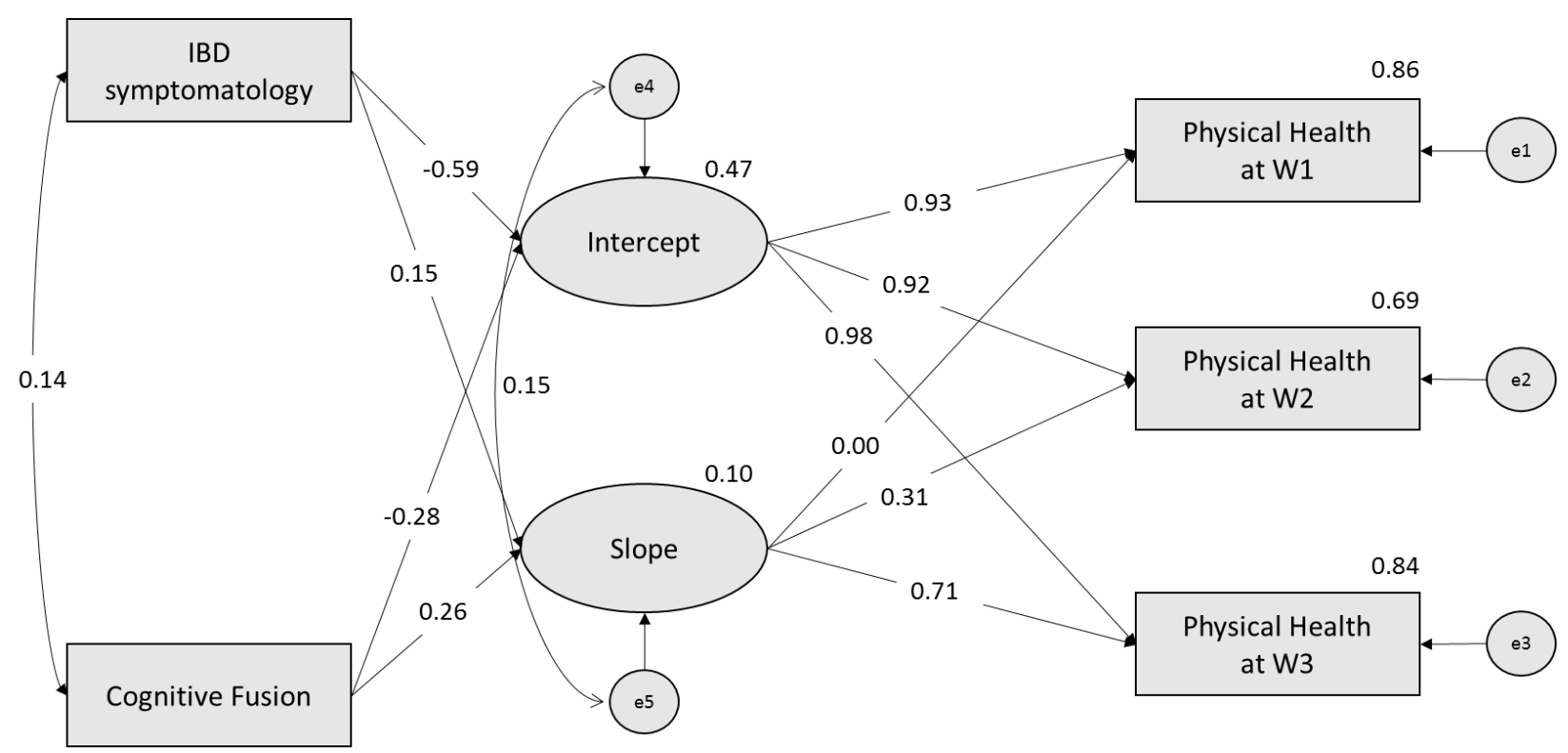

Figure 2. The influence of IBD symptomatology and cognitive fusion in changes in physical health. 